\title{
Rhodamine B Pentyl Ester and its Methyl, Ethyl, Propyl, and Butyl Homologues
}

\author{
Justin A. Ross, ${ }^{*}$ A Benjamin P. Ross, ${ }^{B, C}$ Kelly L. Cosgrove, ${ }^{B}$ Halina Rubinsztein-Dunlop, ${ }^{\text {A }}$ and Ross \\ P. McGeary ${ }^{B, C}$
}

${ }^{A}$ Centre for Biophotonics and Laser Science, School of Physical Sciences;

${ }^{\mathrm{B}} \mathrm{School}$ of Molecular and Microbial Sciences;

$\mathrm{C}_{\text {School of Pharmacy; }}$

The University of Queensland, Brisbane, Queensland 4072, Australia.

Tel.: +61 73366 9583; Fax: +61 73365 1242; E-mail:justinr@physics.uq.edu.au

*Author to whom correspondence should be addressed

Received: 22 June2006 / Accepted: 11 August 2006 / Published: 1 December 2006

Keywords: rhodamine, esterification, acetyl chloride, lipophilicity.

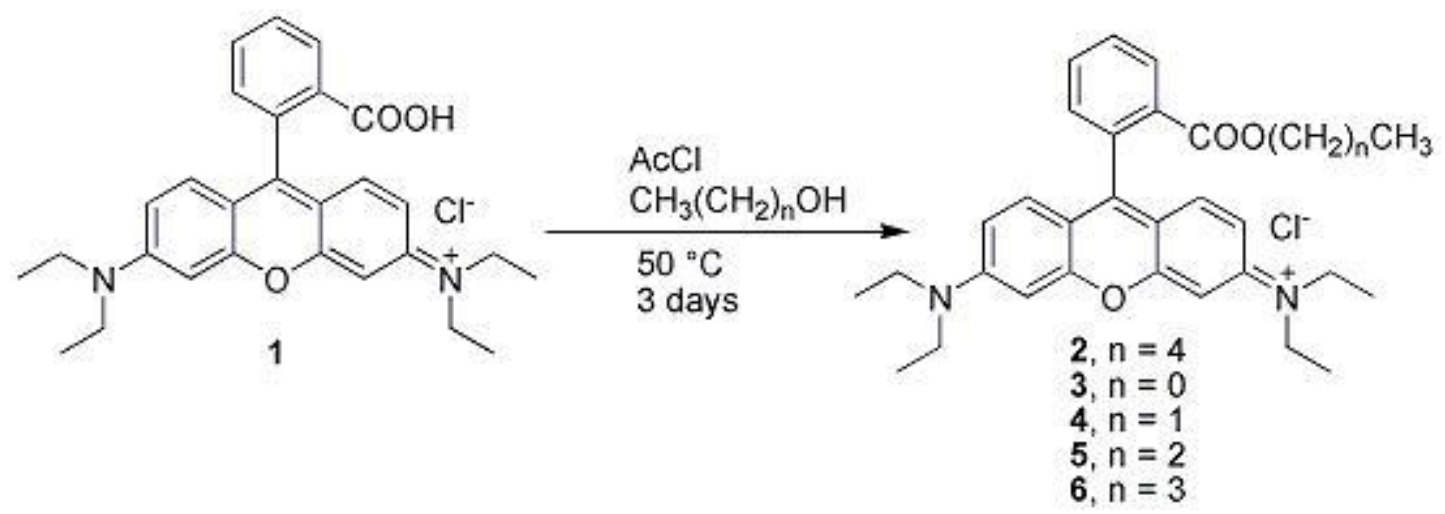

The rhodamines are a highly fluorescent class of compound used in many different fields of research, from the lasing medium in dye lasers to biological stains and markers for cellular drug resistance [1-3]. We are interested in the influence of lipophilicity on the transport of esters of rhodamine in multidrug resistance transporter assays. Herein we describe the synthesis of the novel compound rhodamine B pentyl ester (2), and deposit the spectral data of the known methyl (3), ethyl (4), propyl (5), and butyl (6) homologues [4]. Ester 2 was prepared by a slightly modified facile method [5] that utilizes anhydrous hydrogen chloride generated in situ by the addition of $\mathrm{AcCl}$ to a mixture of the rhodamine $\mathrm{B}$ free acid (1) and $n$-pentanol.

\section{Rhodamine B pentyl ester (2)}

$N$-[6-(Diethylamino)-9-[2-(pentyloxycarbonyl)phenyl]-3H-xanthen-3-ylidene]- $N$-ethylethanaminium chloride

Acetyl chloride $(1.25 \mathrm{~mL}, 17.6 \mathrm{mmol})$ was added dropwise to a stirred mixture of rhodamine B (1, 100 $\mathrm{mg}, 0.21 \mathrm{mmol})$ and $n$-pentanol $(25 \mathrm{~mL})$, and the reaction was heated at $50^{\circ} \mathrm{C}$ under an atmosphere of argon. After 2 days the reaction was $\sim 90 \%$ complete by TLC, and additional acetyl chloride $(250 \mu \mathrm{L}$, $3.52 \mathrm{mmol}$ ) was added to drive the reaction to completion. After a further $24 \mathrm{~h}$ TLC indicated no remaining rhodamine $\mathrm{B}(\mathbf{1})$ and the solution was evaporated in vacuo (water bath temperature $<50{ }^{\circ} \mathrm{C}$ to avoid degradation of the product) to afford rhodamine B pentyl ester (2), which was contaminated with a small amount of $n$-pentanol. The molar ratio of rhodamine B pentyl ester (2): $n$-pentanol was $\sim 3: 1$ by ${ }^{1} \mathrm{H}$ NMR. To separate the ester (2) from residual alcohol, the crude product was dissolved in water $(100 \mathrm{~mL})$ and washed with ethyl acetate $(3 \times 50 \mathrm{~mL})$. Acetonitrile $(35 \mathrm{~mL})$ was added to the aqueous layer and this solution was lyophilised to afford the title compound (2), a hygroscopic amorphous solid (85\%). 
TLC Rf 0.41 (silica gel 60 F254 aluminum sheets, $n$-butanol:water:ethanol 9:2:1).

ES-MS, m/z: $513[\mathrm{M}+\mathrm{H}]^{+}$.

${ }^{1} \mathrm{H}$ NMR (500 MHz, d6-DMSO) d $8.24(1 \mathrm{H}, \mathrm{dd}, J=7.9$ and $1.2 \mathrm{~Hz}), 7.91(1 \mathrm{H}, \mathrm{dt}, J=7.5$ and $1.3 \mathrm{~Hz})$, $7.85(1 \mathrm{H}, \mathrm{dt}, J=7.7$ and $1.3 \mathrm{~Hz}), 7.51(1 \mathrm{H}, \mathrm{dd}, J=7.5$ and $1.1 \mathrm{~Hz}), 7.11(2 \mathrm{H}, \mathrm{dd}, J=9.6$ and $2.4 \mathrm{~Hz})$, $7.02-6.99(4 \mathrm{H}, \mathrm{m}), 3.89(2 \mathrm{H}, \mathrm{t}, J=6.2 \mathrm{~Hz}), 3.65(8 \mathrm{H}$, br q, $J=7.1 \mathrm{~Hz}), 1.20(12 \mathrm{H}$, br t, $J=7.0 \mathrm{~Hz})$ overlapping with $\sim 1.18-1.13(2 \mathrm{H}, \mathrm{m}), 1.06(2 \mathrm{H}$, sextet, $J=7.4 \mathrm{~Hz}), 0.88-0.82(2 \mathrm{H}, \mathrm{m}), 0.71(3 \mathrm{H}, \mathrm{t}, J=7.4$ $\mathrm{Hz})$.

${ }^{13} \mathrm{C}$ NMR (100 MHz [6], d6-DMSO) d 165.1, 157.6, 157.1, 155.1, 133.0, 132.7, 131.0, 130.9, 130.5, $130.4,129.9,114.6,112.9,95.9,65.2,45.3,27.5,27.4,21.7,13.6,12.4$.

UV-visible $\left(\mathrm{H}_{2} \mathrm{O}\right): \lambda_{\max } 559 \mathrm{~nm}$.

HRMS calcd for $[\mathrm{M}+\mathrm{H}]^{+}$513.3117, found 513.3122.

The methyl (3), ethyl (4), propyl (5) and butyl (6) homologues of 2 were synthesized by the same method except that evaporation of the solution in vacuo afforded the ester (3-6) pure, without the need for partitioning between water and ethyl acetate. Previously these compounds were isolated as the bromide, iodide or perchlorate salts, and their spectra were recorded in $\mathrm{CDCl}_{3}$ [4]. Herein we deposit the spectra of the chloride salts (3-6) recorded in $\mathrm{d}_{6}$-DMSO.

\section{Rhodamine B methyl ester (3)}

$N$-[6-(Diethylamino)-9-[2-(methoxycarbonyl)phenyl]-3H-xanthen-3-ylidene]- $N$-ethylethanaminium chloride

TLC R 0.35 (silica gel $60 \mathrm{~F}_{254}$ aluminum sheets, $n$-butanol:water:ethanol 9:2:1).

ES-MS, $m / z: 457[\mathrm{M}+\mathrm{H}]^{+}$.

${ }^{1} \mathrm{H}$ NMR (500 MHz, d6-DMSO) d $8.27(1 \mathrm{H}, \mathrm{dd}, J=7.9$ and $1.2 \mathrm{~Hz}), 7.92(1 \mathrm{H}, \mathrm{dt}, J=7.6$ and $1.3 \mathrm{~Hz})$, $7.84(1 \mathrm{H}, \mathrm{dt}, J=7.7$ and $1.3 \mathrm{~Hz}), 7.51(1 \mathrm{H}, \mathrm{dd}, J=7.5$ and $1.1 \mathrm{~Hz}), 7.08(2 \mathrm{H}, \mathrm{dd}, J=9.6$ and $2.5 \mathrm{~Hz})$, 6.99-6.97 (4H, m), $3.65(8 \mathrm{H}$, br q, $J=7.02 \mathrm{~Hz}), 3.60(3 \mathrm{H}, \mathrm{s}), 1.21(12 \mathrm{H}$, br t, $J=7.0 \mathrm{~Hz})$.

${ }^{13} \mathrm{C}$ NMR (75 MHz, d6-DMSO) d 165.0, 157.9, 157.1, 155.1, 133.4, 133.3, 130.8, 130.8, 130.5, 130.5, $129.2,114.6,112.8,95.9,52.5,45.3,12.5$.

UV-visible $\left(\mathrm{H}_{2} \mathrm{O}\right): \lambda_{\max } 558 \mathrm{~nm}$.

HRMS calcd for $[\mathrm{M}+\mathrm{H}]^{+}$457.2491, found 457.2481.

\section{Rhodamine B ethyl ester (4)}

$N$-[6-(Diethylamino)-9-[2-(ethoxycarbonyl)phenyl]-3H-xanthen-3-ylidene]- $N$-ethylethanaminium chloride

TLC Rf 0.37 (silica gel 60 F254 aluminum sheets, $n$-butanol:water:ethanol 9:2:1).

ES-MS, $m / z: 471[\mathrm{M}+\mathrm{H}]^{+}$.

${ }^{1} \mathrm{H}$ NMR $\left(500 \mathrm{MHz}, \mathrm{d}_{6}-\mathrm{DMSO}\right) \mathrm{d} 8.25(1 \mathrm{H}, \mathrm{dd}, J=7.9$ and $1.1 \mathrm{~Hz}), 7.91(1 \mathrm{H}, \mathrm{dt}, J=7.6$ and $1.4 \mathrm{~Hz})$, 
$7.84(1 \mathrm{H}, \mathrm{dt}, J=7.7$ and $1.2 \mathrm{~Hz}), 7.52(1 \mathrm{H}, \mathrm{dd}, J=7.6$ and $1.1 \mathrm{~Hz}), 7.09(2 \mathrm{H}, \mathrm{dd}, J=9.6$ and $2.5 \mathrm{~Hz})$, 7.01-6.99 (4H, m), $3.98(2 \mathrm{H}, \mathrm{q}, J=7.1 \mathrm{~Hz}), 3.65(8 \mathrm{H}$, br q, $J=7.0 \mathrm{~Hz}), 1.21(12 \mathrm{H}, \mathrm{br} \mathrm{t}, J=7.0 \mathrm{~Hz}), 0.90$ $(3 \mathrm{H}, \mathrm{t}, J=7.1 \mathrm{~Hz})$.

${ }^{13}$ C NMR (75 MHz, d6-DMSO) d 164.7, 157.7, 157.1, 155.1, 133.1, 133.0, 130.9, 130.7, 130.5, 130.4, $129.7,114.6,112.9,95.9,61.0,45.4,13.4,12.4$.

UV-visible $\left(\mathrm{H}_{2} \mathrm{O}\right): \lambda_{\max } 559 \mathrm{~nm}$.

HRMS calcd for $[\mathrm{M}+\mathrm{H}]^{+} 471.2648$, found 471.2643 .

\section{Rhodamine B propyl ester (5)}

$\mathrm{N}$-[6-(Diethylamino)-9-[2-(propoxycarbonyl)phenyl]-3H-xanthen-3-ylidene]- $\mathrm{N}$-ethylethanaminium chloride

TLC Rf 0.39 (silica gel $60 \mathrm{~F}_{254}$ aluminum sheets, $n$-butanol:water:ethanol 9:2:1).

ES-MS, m/z: $485[\mathrm{M}+\mathrm{H}]^{+}$.

${ }^{1} \mathrm{H}$ NMR (500 MHz, d6-DMSO) d $8.25(1 \mathrm{H}, \mathrm{dd}, J=7.8$ and $1.2 \mathrm{~Hz}), 7.91(1 \mathrm{H}, \mathrm{dt}, J=7.6$ and $1.3 \mathrm{~Hz})$, $7.85(1 \mathrm{H}, \mathrm{dt}, J=7.7$ and $1.2 \mathrm{~Hz}), 7.52(1 \mathrm{H}, \mathrm{dd}, J=7.5$ and $1.0 \mathrm{~Hz}), 7.09(2 \mathrm{H}, \mathrm{dd}, J=9.6$ and $2.5 \mathrm{~Hz})$, $7.02-6.99(4 \mathrm{H}, \mathrm{m}), 3.89(2 \mathrm{H}, \mathrm{t}, J=6.4 \mathrm{~Hz}), 3.65(8 \mathrm{H}$, br q, $J=7.0 \mathrm{~Hz}), 1.29(2 \mathrm{H}$, sextet, $J=6.9 \mathrm{~Hz}), 1.20$ $(12 \mathrm{H}$, br t, $J=7.0 \mathrm{~Hz}), 0.62(3 \mathrm{H}, \mathrm{t}, J=7.4 \mathrm{~Hz})$.

${ }^{13} \mathrm{C}$ NMR (75 MHz, d6-DMSO) d 164.9, 157.7, 157.1, 155.1, 133.1, 132.9, 130.9, 130.8, 130.5, 130.4, $129.8,114.6,112.9,95.9,66.7,45.4,21.1,12.4,10.0$.

UV-visible $\left(\mathrm{H}_{2} \mathrm{O}\right): \lambda \max 559 \mathrm{~nm}$.

HRMS calcd for $[\mathrm{M}+\mathrm{H}]^{+} 485.2804$, found 485.2801 .

\section{Rhodamine B butyl ester (6)}

$N$-[6-(Diethylamino)-9-[2-(butoxycarbonyl)phenyl]-3H-xanthen-3-ylidene]- $N$-ethylethanaminium chloride

TLC $R_{f} 0.40$ (silica gel $60 F_{254}$ aluminum sheets, $n$-butanol:water:ethanol 9:2:1).

ES-MS, m/z: $499[\mathrm{M}+\mathrm{H}]^{+}$.

${ }^{1} \mathrm{H}$ NMR (500 MHz, d6-DMSO) d $8.24(1 \mathrm{H}, \mathrm{dd}, J=7.9$ and $1.2 \mathrm{~Hz}), 7.91(1 \mathrm{H}, \mathrm{dt}, J=7.5$ and $1.4 \mathrm{~Hz})$, $7.84(1 \mathrm{H}, \mathrm{dt}, J=7.7$ and $1.4 \mathrm{~Hz}), 7.51(1 \mathrm{H}, \mathrm{dd}, J=7.6$ and $1.1 \mathrm{~Hz}), 7.10(2 \mathrm{H}, \mathrm{dd}, J=9.6$ and $2.5 \mathrm{~Hz})$, $7.02-7.00(4 \mathrm{H}, \mathrm{m}), 3.91(2 \mathrm{H}, \mathrm{t}, J=6.2 \mathrm{~Hz}), 3.65(8 \mathrm{H}, \mathrm{br} \mathrm{q}, J=7.2 \mathrm{~Hz}), 1.21(12 \mathrm{H}, \mathrm{br} \mathrm{t}, J=7.0 \mathrm{~Hz})$ overlapping with $\sim 1.20-1.15(2 \mathrm{H}, \mathrm{m}), 0.94(2 \mathrm{H}$, sextet, $J=7.6 \mathrm{~Hz}), 0.68(3 \mathrm{H}, \mathrm{t}, J=7.4 \mathrm{~Hz})$.

${ }^{13} \mathrm{C}$ NMR (75 MHz, d6-DMSO) d 165.0, 157.5, 157.1, 155.1, 133.0, 132.7, 130.9, 130.7, 130.4, 130.3, $129.8,114.6,112.8,95.8,64.9,45.3,29.8,18.4,13.4,12.4$.

UV-visible $\left(\mathrm{H}_{2} \mathrm{O}\right): \lambda_{\max } 559 \mathrm{~nm}$.

HRMS calcd for $[\mathrm{M}+\mathrm{H}]^{+}$499.2961, found 499.2973. 
We thank Mr. Graham MacFarlane (School of Molecular and Microbial Sciences, The University of Queensland) for accurate mass measurements. This work was supported by funds from the Centre for Biophotonics and Laser Science, School of Physical Sciences, The University of Queensland.

\section{References}

1. Zollinger, H. Color Chemistry : Syntheses, Properties, and Applications of Organic Dyes and Pigments, 2nd rev. ed.; VCH: Weinheim, 1991.

2. Gordon, P. F.; Gregory, P. Organic Chemistry in Colour; Springer-Verlag: New York, 1983.

3. Abrahart, E. N.; Dyes and Their Intermediates, 2nd ed.; Edward Arnold: London, 1977.

4. Ramos, S. S.; Vilhena, A. F.; Santos, L.; Almeida, P. Magn. Reson. Chem. 2000, 38, 475-478.

5. Ross, J. A.; Ross, B. P.; Rubinsztein-Dunlop, H.; McGeary, R. P. Synth. Commun. 2006, 36, 1745-1750.

6. The ${ }^{13} \mathrm{C}$ NMR spectra of 3-6 were run at $75 \mathrm{MHz}$ whereas the ${ }^{13} \mathrm{C}$ NMR spectrum of 2 was recorded at $100 \mathrm{MHz}$ to enable resolution of the resonances which appeared at 27.46 and $27.43 \mathrm{ppm}$ in the $100 \mathrm{MHz}$ spectrum.

(C) 2006 MDPI. All rights reserved. 